\title{
Die Behandlung traumatischer Weichteilwunden mit der Vakuumtherapie - Grundlagen, Indikationen und klinische Anwendung
}

\author{
Kirsten Sommer, Tobias Hüfner, Christian Krettek
}

\section{Zusammenfassung}

Das Management komplexer Weichteilverletzungen stellte schon immer eine Herausforderung an den Chirurgen dar. Die Vakuumtherapie akuter und chronischer Wunden ist eine effiziente und breitflächig angewandte Alternative der Wundbehandlung. Die klinische Erfahrung der letzten 2 Jahrzehnte sowie einige Tier- und In-vitroStudien beschreiben die potenzielle Überlegenheit der Vakuumtherapie gegenüber der konventionellen Wundbehandlung, obwohl nur rudimentäre Beweise für die tatsächliche Überlegenheit vorliegen. Die potenziellen Effekte der Vakuumtherapie werden auf den kontinuierlichen Abtransport des Wundsekrets, die Anregung der Granulationsbildung und die Wundretraktion aufgrund des Unterdrucks zurückgeführt. Weitere Vorteile sind die leichte Handhabung, der Patientenkomfort und die hygienische Abschirmung der Wunde gegen Kontaminationen.
Treatment of Traumatic Soft Tissue Injury with Vacuum Therapy Background, Indications and Clinical Implementation

The management of complex soft-tissue injuries remains a challenge for the surgeon. The treatment of acute and chronic wounds with the vacuum-assisted-closure technique (VAC) is an efficient and widely accepted alternative for wound management. The clinical experience of the last two decades, as well as a number of animal and in-vitro-studies describe the potential advantages of this method compared to conventional wound treatment, even though the evidence for its superiority is only weak. The potential effects of VAC therapy are attributed to (1) continuous removal of wound fluid and debris, (2) the stimulation of the formation of granulation tissue, and (3) wound retraction due to negative pressure. Other advantages are the easy handling, the patient comfort and the hygienic shielding of the wound against environmental contamination.

\section{Einleitung}

Bei Knochen- und Gelenkverletzungen spielt der begleitende Weichteilschaden eine erhebliche Rolle und bestimmt im weiteren Verlauf die Prognose des Heilungsprozesses. Haut, Unterhaut, Muskulatur, Sehnen, Blutgefäße und Nerven können sowohl von außen wie auch von innen in unterschiedlichem Ausmaß geschädigt werden. Dies schnellstmöglich und vollständig zu erkennen und in das Behandlungsregime mit einzubeziehen,

OP-JOURNAL 2009; 25: 170-175

(c) Georg Thieme Verlag KG Stuttgart · New York DOI http://dx.doi.org/10.1055/s-0029-1186275 erfordert viel Erfahrung und den Einsatz des gesamten Spektrums der konservativen und operativen Möglichkeiten.

Patienten mit problematischen Wunden stellen eine signifikante Bürde für das Gesundheitswesen dar. Die Weichteilschädigung der Extremitäten repräsentiert einen der wichtigsten Risikofaktoren für Amputationen, Sepsis und die stationäre Aufenthaltsdauer. Aus Sicht des Patienten ist die Wundtherapie, auch ohne verstümmelnde Operationen, oft schmerzhaft und langwierig.

Moderne Wundheilungskonzepte beinhalten verschiedene Arten von Wundauflagen und die topische Applikation diverser Substanzen, obwohl nur wenige dieser Behandlungsmodalitäten überzeugend gezeigt haben, dass sie höhere Wundheilungsraten erzielen als die traditionelle feuchte Kompressenbehandlung $[3,19]$.

Das unter den Synonymen „topical negative pressure“ (TNP), „subatmospheric pressure“ (SAD), „vacuum sealing technique“ (VST), „sealed surface wound suction“ (SSS) und „vacuum assisted closure“ (VAC) bekannte Therapiekonzept der Vakuumtherapie ist eine revolutionäre Methode zur Behandlung akuter und chronischer Wunden, die Ende der 80erJahre entwickelt wurde und seit Anfang der 90er-Jahre in zunehmendem Ausmaß in allen chirurgischen Disziplinen eingesetzt wird. Der breite Einsatz der Vakuumtherapie in der Traumatologie ist heute fest etabliert. Die Therapie soll aber nicht die chirurgisch korrekte Behandlung von Weichteilverletzungen ersetzen, vielmehr ist sie als temporärer Ersatz für den entstandenen Defekt und zur Verbesserung der Wundkonditionierung zu sehen [15].

Als wichtigste klinisch-relevante Vorteile der Vakuumtherapie gelten nachfolgende Effekte auf die Wundheilung und das betroffene Gewebe, die als Ergebnis des gleichmäßig auf die gesamte Wundoberfläche einwirkenden Soges angesehen werden. Die beschriebenen Effekte an der Wunde sind u.a. die Wundretraktion, das Anregen der Bildung von Granulationsgewebe, die Fortsetzung der effektiven Wundreinigung nach PrimärDébridement und die druckbedingte Minderung eines interstitiellen Ödems mit konsekutiver Verbesserung der Mikrozirkulation $[4,6,15,21]$.

\section{Das Prinzip und die Technik der Vakuumtherapie}

Physikalisch betrachtet entsteht ein Unterdruck, sobald der wirkende Luftdruck unterhalb des atmosphärischen Druckes liegt. Auf Meereshöhe und bei Raum- 
temperatur beinhaltet ein bestimmtes Volumen von Luft Moleküle, die sich in unterschiedliche Richtungen bewegen. Von diesen Molekülen geht eine Kraft aus, die dem normalen atmosphärischen Druck von $760 \mathrm{mmHg}$ entspricht. Werden nun Moleküle mittels Saugpumpe aus einem bestimmten abgeschlossenen Bereich (z. B. Wunde) entfernt, wird Unterdruck erzeugt.

Die Pioniere auf dem Gebiet der Vakuumforschung, Morykwas und Argenta, starteten eine Tierversuchsreihe, in der sie die Vakuumtherapie in Verbindung mit einem Polyurethan-(PU-)Schaumverband einsetzten. Hierbei diente der PU-Schaum als Verbindung zwischen der Wundoberfläche und der Vakuumquelle [15]. Dieser PU-Schaum war das entscheidende Element für die Entwicklung eines wirtschaftlich nutzbaren Systems durch die Firma Kinetic Concepts Inc. (VAC-System, Fa. KCI, San Antonio, Texas). Diverse Systeme sind heute für die Vakuumversiegelung von Wunden verfügbar. Als eines der einfachsten Systeme der Vakuumtherapie stellen derzeit die klassischen Redon-Drainagen als epifasziale Wund-Saugdrainagen bei „wasserdichtem" Wundverschluss dar. Diese Vakuumapplikation ist jedoch nicht kontrollierbar und verursacht Spitzendrücke von bis zu 900 mmHg. Die Markteinführung der V.A.C.-Therapie der Firma KCI im Jahr 1995 konnte dieses Defizit beheben. Dieses System besteht aus einem schwarzen, hydrophoben PU-Schwamm mit offenen Poren (400-600 $\mu \mathrm{m})$, welcher in der Wunde, abschließend mit den Wundrändern, platziert wird. Alternativ ist die Verwendung eines weißen, hydrophilen Polyvinylalkohol-(PVA-)Schwammes, mit dichteren, kleineren Poren (1$200 \mu \mathrm{m})$ möglich.

Die Auswahl des Schwammes hängt von der Wundsituation und dem Behandlungsziel ab.

Die regelmäßige Struktur und Verteilung der Poren ermöglichen die gleichmäßige Verteilung des Druckes über die gesamte Wundoberfläche. Eine wasserdampfdurchlässige, transparente und keimdichte Polyurethanklebefolie wird über den Schwamm und die Wundränder auf die gesunde Haut geklebt. Sie schließt die Wunde einerseits hermetisch ab und modelliert durch den angesetzten Sog den Schwamm in den Wunddefekt. Zur Erzeugung des Vakuums dient ein „Saugnapf“ (trac-pad), der mittels eines Drainageschlauchs in Verbindung mit einer
Tab. 1 Komponenten des Vakuumsystems.

In Abhängigkeit von der Wundsituation und dem Behandlungsziel verwendet man:

- einen schwarzen, hydrophoben PUSchwamm oder einen weißen, hydrophilen PVA-Schwamm

- eine transparente semiokklusive Klebefolie zum luftdichten Versiegeln der Wunde

- einen nicht kollabierbaren Drainageschlauch, der den Schwamm mit der Vakuumquelle verbindet

einen Auffangkanister für das Wundexsudat

- eine Vakuumquelle

Vakuumquelle steht. Dieses Pad wird über ein kleines Loch in der Folie geklebt, welches über dem Schwamm eingeschnitten wird, und mit einer elektrisch betriebenen Therapieeinheit verbunden, welche das Vakuum generiert. Ein Mikroprozessor verarbeitet die Eingänge des Systems und alarmiert, wenn der eingestellte Drucklevel abweicht. Bei angelegtem Vakuum zieht sich der Schaum zusammen, was zur Wundkontraktion und Exsudatdrainage führt [6,15] (Tab.1). Derzeit gibt es keine vergleichbare effiziente Lösung am Markt als das KCIV.A.C.-System, jedoch finden sich für die Unterdruckapplikation verschiedene Vakuumquellen ohne CE-Zertifizierung in der klinischen Anwendung. So werden Redon-Flaschen, aber auch einfache Wandabsaugungen als nutzbare Vakuumquellen beschrieben $[2,7,8]$.

Die Vakuumtherapie dient bei den akuten, in der Regel posttraumatischen oder postoperativen Wunden in erster Linie als Methode zur temporären Deckung und/oder Konditionierung von Wunden bis zum definitiven Verschluss durch Sekundärnaht oder plastischen Eingriff (Spalthauttransplantation, Lappenplastik).

Für die Durchführung der Vakuumtherapie stehen heute über 30 verschieden geformte und konfektionierte Schwämme aus 2 unterschiedlichen Materialien (Polyvinylalkoholschwamm und Polyurethanschwamm) zur Verfügung. Für einzelne Indikationen sind spezielle Schwammsysteme entwickelt worden (z.B. Behandlung des offenen Abdomens, Fersenulkus, Verbrennungen der Hand) (Tab. 2).
Tab. 2 Indikationen der Vakuumtherapie.

- akute und chronische Weichteilschäden

\begin{tabular}{l}
\hline - infizierte Wunden \\
\hline - Wunden nach offenen Frakturen \\
\hline $\begin{array}{l}\text { nässende chirurgische Wunden } \\
\text { (Sekretion }>48 \mathrm{~h})\end{array}$ \\
\hline $\begin{array}{l}\text { - Wunden mit freiliegenden Sehnen, } \\
\text { Implantaten oder offenen Gelenken }\end{array}$ \\
\hline - Wunden nach einer Dermatofasziotomie \\
bei Kompartmentsyndrom \\
\hline - chronische Wunden (>3 Monate) \\
\hline - Wunden mit schwierigem Wundver- \\
schluss, die primär offen gelassen werden \\
\hline - offenes Abdomen \\
\hline - sternale Wunden \\
\hline - Fixierung von Spalthauttransplantaten \\
\hline - Verbrennungen
\end{tabular}

Vor Beginn einer Vakuumtherapie müssen saubere chirurgische Wundverhältnisse geschaffen werden.

Dafür ist unter Umständen ein chirurgisches Débridement der Wunde notwendig. Der Vakuumverband kann zwischen 2 und 7 Tagen verbleiben (abhängig von der Wunde, der Keimbesiedelung und dem gewählten Schwammmaterial). Der Vakuumschwammwechsel kann in Abhängigkeit vom Wundschmerz, dem technischem Aufwand, sowie den Anforderungen an die Sterilität unter stationären Bedingungen, im Operationssaal oder unter ambulanten Bedingungen durchgeführt werden.

Die Dauer der Behandlung richtet sich nach dem Erfolg der Wundheilung. Sie kann als Interimstherapie zur Konditionierung der Wunde wenige Tage bis wenige Wochen oder als Definitivtherapie bis zum Verschluss der Wunde andauern.

Die Vakuumtherapie ermöglicht die Anpassung diverser Parameter an die jeweilige Wundsituation. Veränderbar sind das Schwammmaterial, die Sogstärke (wählbar sind Werte zwischen 50 und $200 \mathrm{mmHg}$ ), der Sogmodus (intermittierend oder kontinuierlich), die Vakuumquelle, sowie die optionale Medikamententherapie (Silberbeschichtung, topische Antibiotikatherapie oder Applikation von Wachstumsfaktoren).

In der Frühphase des Einsatzes der Vakuumtherapie zeigte sich überraschend, 
dass diese Therapieform über eine ausgeprägte Potenz zur Granulationsgewebeneubildung verfügt. In einzelnen Situationen der Extremitätenchirurgie, in denen freiliegende Knochen, Sehnen und Metallimplantate nicht durch plastisch-chirurgische Eingriffe gedeckt werden können, ist die Vakuumtherapie die Methode der Wahl, um dennoch eine Deckung dieser Strukturen zu erreichen. Die Ursachen dieser überraschenden Potenz zur Granulationsgewebeneubildung sind bislang nicht geklärt, jedoch liefern einige Studien Hinweise für die pathophysiologischen Vorgänge $[4,5,17,18]$.

Chen et al. wies in einer Tierstudie nach, dass die V.A.C.-Therapie die Blutzirkulation in Wunden bedeutend verbessert. Dies wird u.a. durch die Erhöhung des Durchmessers der Kapillaren, ein erhöhtes Blutvolumen, eine Stimulation endothelialer Zellproliferation und Angiogenese, einem verringerten endothelialen Abstand und eine Wiederherstellung der Integrität kapillärer Basalmembranen verursacht [4]. Andere Studien postulieren, dass mechanischer Stress durch das Vakuum die Sekretion von angiogenetischen Faktoren erhöhen könnte. Auch die Hautperfusion scheint günstig von der Vakuumtherapie beeinflusst zu werden und bedingt eine Reduktion der Ödementwicklung. Zusätzlich kann sie eine Hyperperfusion bei 2-gradigen Verbrennungswunden induzieren, falls die Therapie innerhalb von 6 Stunden nach Trauma begonnen und für mindestens 48 Stunden durchgeführt wird. Um jedoch die wundnahe Perfusion nicht zu sehr einzuschränken, sollte vor allem im subkutanen Gewebe der Sog nicht zu hoch eingestellt werden. Die intermittierende Therapie kann zu einem Anstieg der Durchblutung führen und eine andauernde Ischämie des Gewebes verhindern $[4,5,9,14,17,18,20]$.

Frühere Studien ergaben widersprüchliche Angaben bezüglich der bakteriellen Belastung von Wunden unter Vakuumtherapie. Eine signifikante Abnahme der Bakterienzahl wurde in Gewebeproben bei Schweinen unter dieser Therapie verzeichnet, wie auch einige klinische Studien eine Abnahme der Bakterienlast beschreiben $[2,8,14,22]$. Im Gegensatz zu diesen Beobachtungen konnten Moues et al. keinen Unterschied der Biolast zwischen vakuum- und gazebehandelten Wunden feststellen [16]. Weed beobachte sogar einen Anstieg der Bakterienlast während der V.A.C.-Therapie [22].

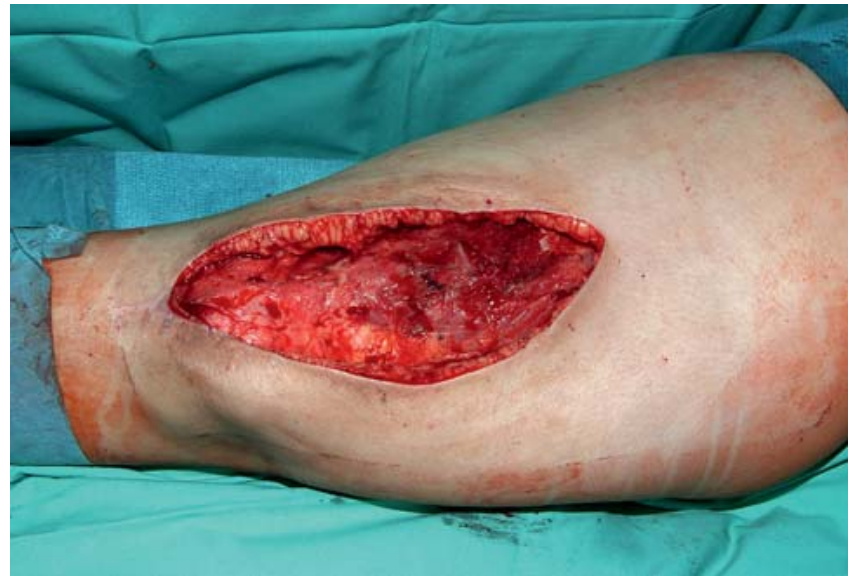

Abb. 1 Infizierte Wunde des Oberschenkels rechts nach Abszessspaltung, Débridement und Spülung der Wunde.

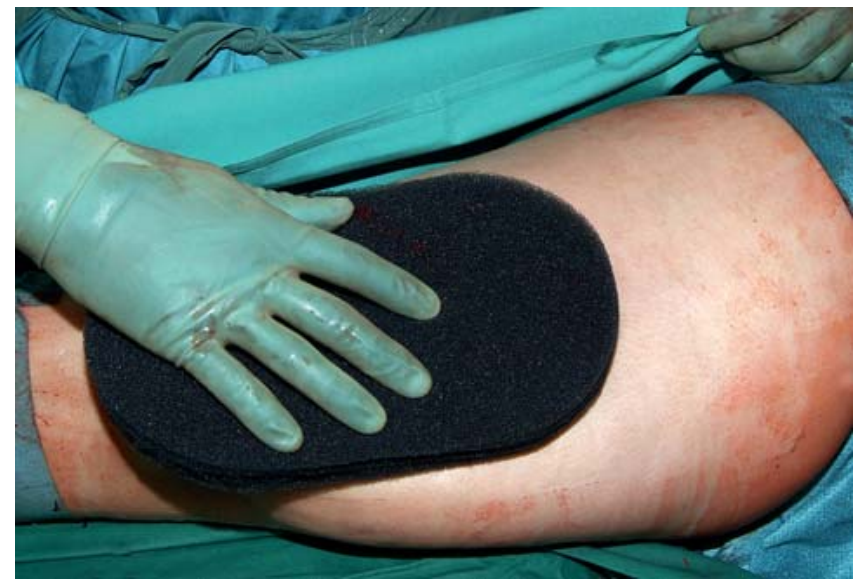

Abb. 2 Anpassen des Schwammes an die Wundform (schwarzer PUSchwamm).

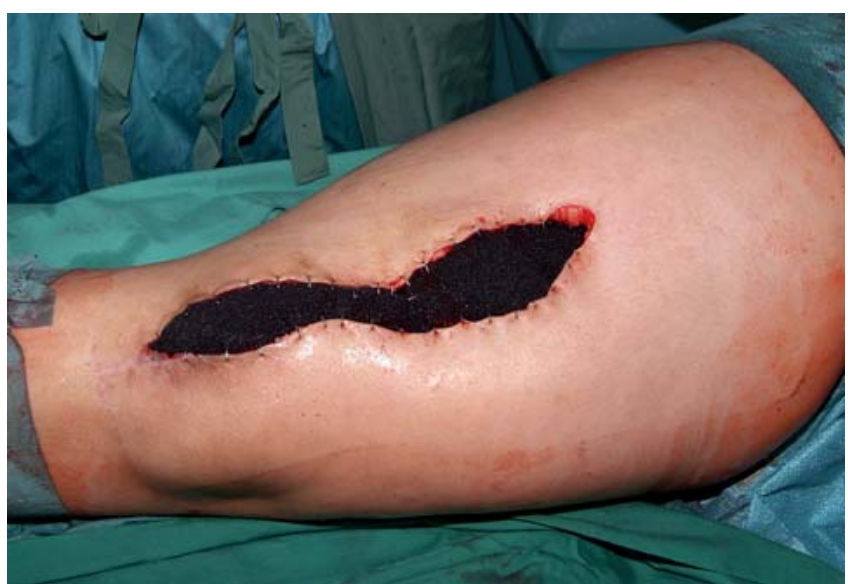

Abb. 3 Fixierung des Schwammes an den seitlichen Wundrändern mit Hautklammern (in anderen Fällen kann dies auch mittels Hautnaht oder ohne jegliche Fixierung erfolgen).

\section{Anwendungsbeispiel}

Anhand einer exemplarischen Darstellung eines Patientenfalls sollen die einzelnen Schritte der Vakuumtherapie vorgestellt werden. Der Fall zeigt die hygienische und komfortable Versorgung einer infizierten Oberschenkelwunde einer 22jährigen Patientin (Abb. 1-8).

\section{Indikationen, Kontraindikationen und Komplikationen}

Das Therapiekonzept der Vakuumtherapie findet heute ein großes Einsatzgebiet in der Behandlung von chronischen und infizierten Wunden, Verbrennungen sowie plastischen Eingriffen. So etablierte sich die V.A.C.-Therapie in der Herzchirurgie bei sternalen Wundinfektionen zur Methode der Wahl. Weiterhin fungiert sie heute als praktikable Alternative des temporären Verschlusses des offenen 

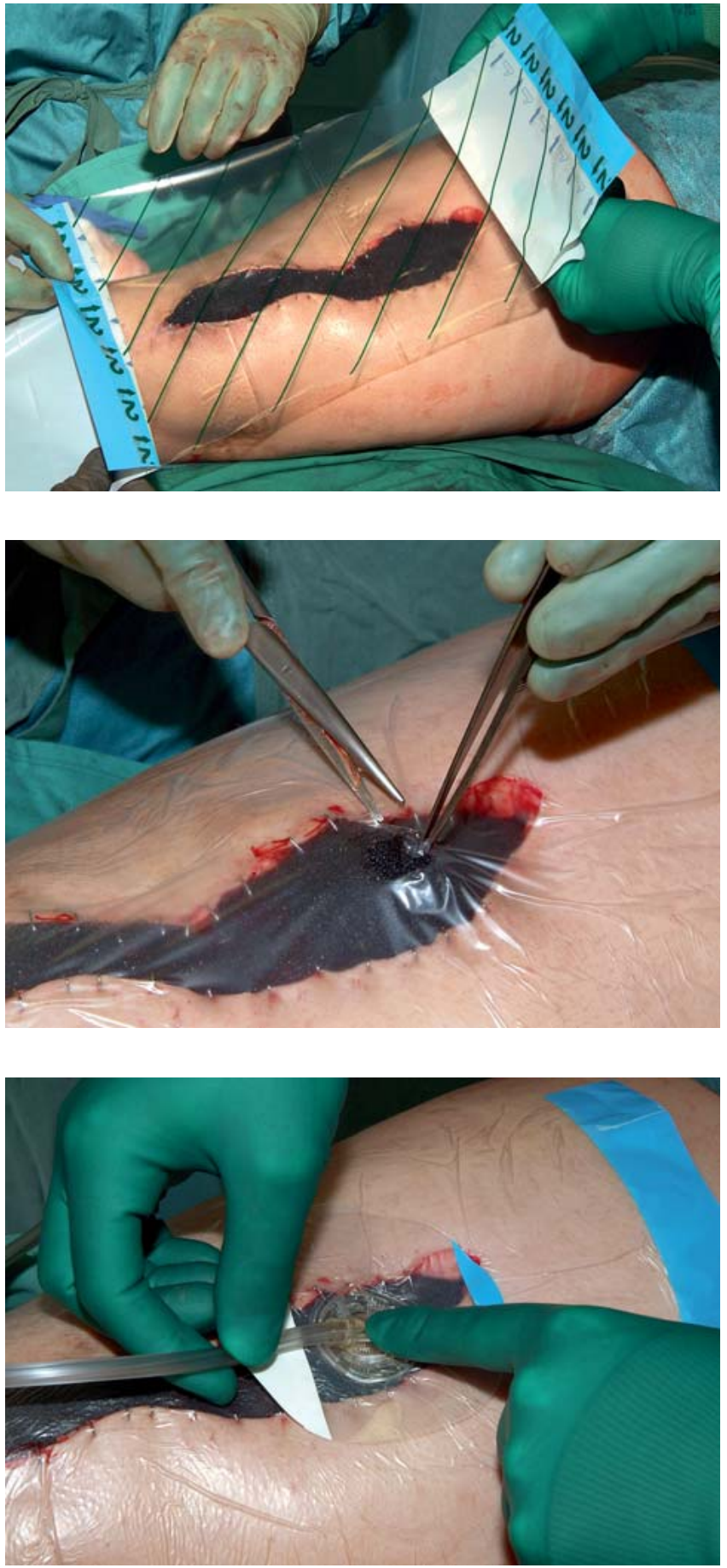

Abdomens, zur Fixierung von Hauttransplantaten, der Behandlung verschmutzter Wunden, sowie penetrierender Weichteilverletzungen, Décollement-Verletzungen, offener Wunden nach Fasziotomien bei Kompartmentsyndrom, offenen Frakturen und Amputationsverletzungen. Bei den chronischen Wunden bewährte sich die Vakuumtherapie beim Dekubitus, Ulcus cruris und vor allem beim diabetischen Fußsyndrom. Einige weitere Indikationen wie enterkutane und lymphokutane Fisteln werden von
Abb. 5 Einschneiden eines kleinen Loches in die Folie.

Abb. 6 Aufkleben des Trac-Pads auf die Folie.

Abb. 4 Versiegelung der Wunde mitparenter Klebefolie.

einigen Autoren ebenfalls als Indikationen angesehen [6,12,14,18, 19,21,24,25].

Es muss jedoch noch einmal betont werden:

V. A. C.-Therapie ersetzt nicht das radikale chirurgische Wunddébridement und die Antibiotikatherapie. VAC ist ebenfalls keine Infekttherapie, sondern ein inzwischen wichtiges Behandlungsmodul im Rahmen des chirurgischen Therapiekonzepts. tels luftdichter trans-
Die Vielfalt der heute verfügbaren Schwämme erlaubt einen zunehmenden differenzierten Einsatz, der der jeweiligen Indikation optimal angepasst werden kann. In Einzelfällen gibt es speziell konfektionierte Schwämme, die exakt für eine Indikation bzw. Lokalisation angeboten werden. Während bei den Schwämmen beinahe die gesamte Palette von einem Hersteller angeboten wird, stehen dem Anwender bei den „Sogerzeugern“ die verschiedensten technischen Lösungen zur Verfügung. Diese unterscheiden sich in der Anwenderfreundlichkeit und in der Praktikabilität unter stationären und ambulanten Bedingungen erheblich. Wesentliche Unterschiede bestehen hinsichtlich der Möglichkeit, Fehlfunktionen zu erkennen und $\mathrm{zu}$ melden (Alarmfunktion). Nur ein Hersteller (KCI) bietet Geräte an, in denen diese Alarmfunktion für Sogverlust und Kanisterüberfüllung integriert ist.

Die Vorzüge des V.A.C.-Systems zeigen sich vor allem durch den sterilen, geschlossenen Verband, welcher eine feuchte Wundheilungsumgebung erzeugt.

Er stellt jedoch keinen Okklusivverband dar, da das Wundexsudat permanent absaugt wird. Neben den lokalen sich günstig auswirkenden Wirkungsmechanismen auf zellulärer Ebene sind bei korrekter Anwendung der Vakuumtherapie das mechanische Drainageprinzip und die Verkleinerung von Toträumen in den Wunddefekten wichtige Hilfsmittel zur Reduktion der bakteriellen Besiedlung und Infektbehandlung offener Wunden. Vorsicht ist bei der Anwendung der Methode dort geboten, wo in akuten Traumasituationen aufgrund der Wundlokalisation oder systemisch bestehender Gerinnungsstörung eine Blutung provoziert und unterhalten werden kann [24]. Ebenfalls darf eine V.A.C.-Therapie nicht bei Liquorfistel erfolgen. Nerven, Sehnen und Gefäße sollten entweder muskelgedeckt sein oder durch eine artifizielle zusätzliche Maßnahme (z. B. Fettgaze) protektiv abgedeckt werden (Tab. 3).

Bei einigen Wunden ist die Applikation des Vakuumsystems unter nicht sterilen Kautelen möglich. Nach der Wahl der geeigneten Größe des Schaumverbands wird dieser in der an die jeweilige Wundgröße angepassten Form zugeschnitten. Wichtig ist hierbei die genaue Anpassung des Schaumverbands an die Wund- 

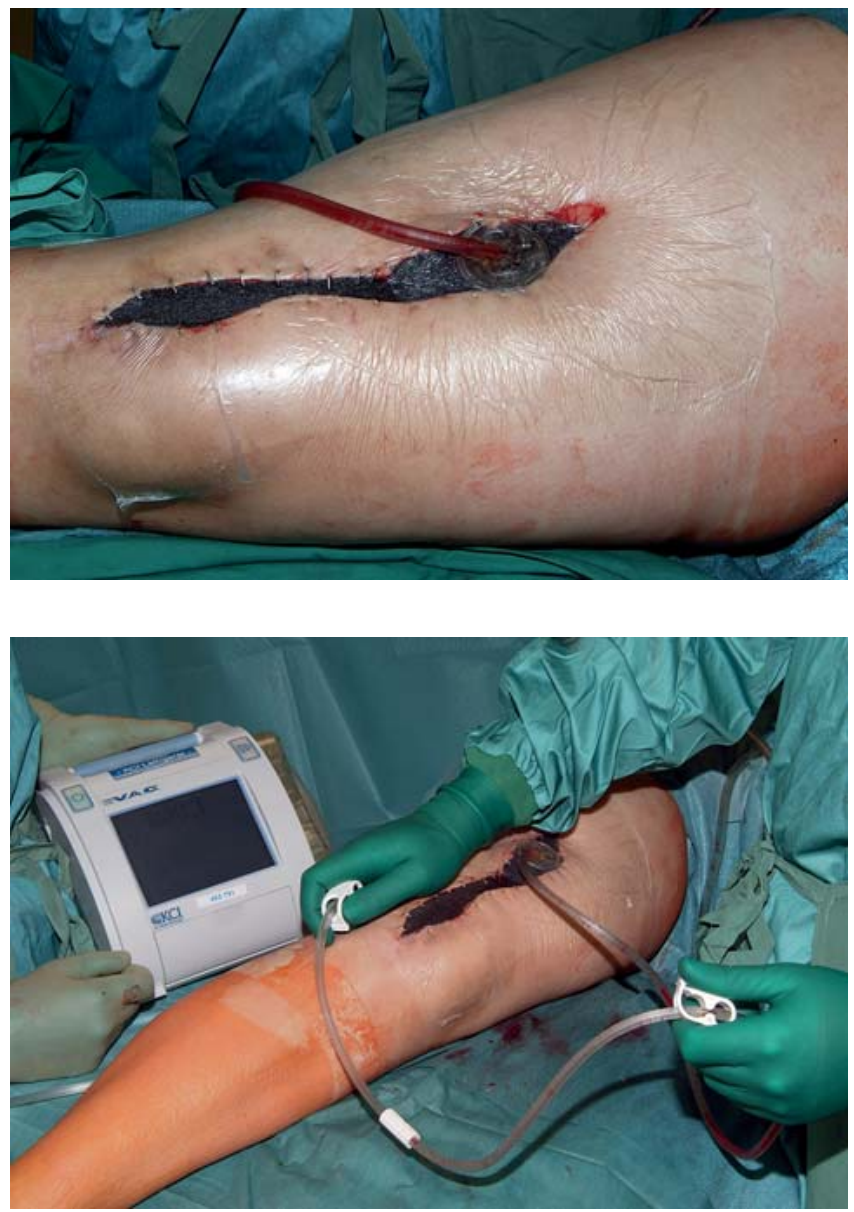

oberfläche, sodass dieser an der gesamten Wundfläche anliegen kann. Falls ein direkter Kontakt mit der Wundfläche, insbesondere in der Tiefe, nicht erreicht wird, ist eine „Totraumversiegelung“ und die Entwicklung einer Abszessbildung möglich.

Das Ziel der Vakuumtherapie sollte daher sein, Toträume zu vermeiden und die Wundheilung aus der Tiefe zu fördern.

Bei Bedarf kann für eine vollständige Versiegelung der gesamten Wundoberfläche der Schaumverband in mehrere Teile zerschnitten werden. Wichtig dabei ist, dass der Schaumverband locker in die Wunde gelegt wird. Ein „packing“ im Sinne einer Tamponade ist zu vermeiden. Ebenso sollte das Schwammmaterial nicht über die Wundränder hinaus auf die unverletzte Haut aufgebracht werden, da die Gefahr der Mazeration und Kontaktdermatitis besteht [21].

Bei lang dauernder V.A.C.-Therapie kann durch sukzessive Unterschneidung des Schwammes die Wundfläche durch Hauttraktion verkleinert werden.
Abb. 8 Aufgrund der kompakten Vakuumquelle kann der Patient frühzeitig mobilisiert werden.

Abb. 7 Nach Anschluss des Vakuums Im Vergleich zum Ausgangsbefund (Abb. 6) zeigt sich durch das sogbedingte „Schrumpfen" des Schwammes eine deutliche Verschmälerung der Wunde.

Bei großen, ausgedehnten Wunden ist ein Verbandswechsel im Operationssaal unter sterilen Kautelen in Analgosedierung erforderlich. Bei kleineren Wunden kann für einen Verbandwechsel auf eine Anästhesie verzichtet werden oder aber ein Lokalanästhetikum in den Schwamm gegeben werden, nachdem der Sog entfernt wurde.

Als Kontraindikation für die Vakuumverbandapplikation sind die akute Blutung und Blutungsgefahr unbestritten. Auch freiliegende Gefäße und Gefäßanastomosen, ein nekrotischer Wundgrund, eine nicht behandelte Osteomyelitis sowie Wunden in neoplastischem Gewebe sollten nicht mit der Vakuumtherapie behandelt werden $[1,11,23,24]$ (Tab. 4).

Bislang wurden nur wenige Einzelberichte über Nebenwirkungen und Zwischenfälle im Rahmen der Vakuumtherapie publiziert. Von besonderer klinischer Relevanz sind hierbei das Auftreten eines Toxic Shock Syndromes infolge unbemerkten Sogverlusts, die Entstehung einer Wundinfektion durch Aerobier während der Vakuumtherapie und Blut- bzw. Flüssigkeitsverluste aufgrund mit $125 \mathrm{mmHg}$.
Tab. 3 Vorteile der Vakuumtherapie.

\begin{tabular}{l} 
- Reduzierung der Fistelbildung \\
\hline - erhöhte Chance des primären Wundver- \\
schlusses
\end{tabular}

Tab. 4 Kontraindikationen bei der Behandlung mit Vakuumtherapie.

- direkte Platzierung des Schwammes auf
Gefäßen, parenchymatösen Organen
oder Sehnen

unkontrollierter Absaugung $[1,10,15$, 20]. Die häufigsten Nebenwirkungen der Vakuumtherapie sind Schmerz durch Einwirken des Soges, Schmerzen beim Verbandwechsel und Mazeration sowie Druckschäden des an die Wunde angrenzenden Hautareals $[1,5,11,13,20,24]$.

\section{Fazit}

Da die Vakuumtherapie in Verbindung mit dem V.A.C.-System eine verhältnismäßig teure Investition darstellt, ist ein Vergleich mit den konventionellen Heilungsmethoden empfehlenswert. Die Anschaffungskosten für Folien, Schläuche und Kanister sowie die Mietkosten für die Vakuumpumpe liegen um einiges höherer als die Kosten einer Behandlung mit feuchten Kompressen. Diese Kosten machen jedoch nur einen geringen Teil der Gesamtkosten aus, die für die Behandlung von Wunden nötig sind. Diese Kosten beinhalten u.a. Schwestern-/ Arztarbeitszeit, Krankenhausaufenthalte 
und Kosten möglicher Folgeerkrankungen. Die ökonomische Evaluierung zweier unterschiedlicher Behandlungsmethoden, wie zum Beispiel der Vergleich zwischen Vakuumtherapie und konventionellen Behandlung, zielt darauf ab, deren relative Kosten und Nutzen darzustellen. Klinische Studien mit hohem Evidenzlevel zeigen, dass sowohl ökonomische als auch klinische Vorteile der Vakuumtherapie für die Anwendung sprechen. Einsparungen im Bereich der schnelleren Heilungsraten, des reduzierten Verbandwechsels sowie in Bezug auf den Krankenhausaufenthalt und die Pflegeleistung als auch die Reduzierung von Folgeerkrankungen, relativieren die höheren Anschaffungskosten.

Die Reduzierung der Verbandwechsel, der Krankenhausaufenthalte und -einweisungen und der Rezidive machen die Vakuumtherapie trotz höherer Tagesbehandlungskosten zu einer deutlich effizienteren und patientenfreundlicheren Behandlungsmethode.

Die Vakuumtherapie kann aber nur als ergänzende therapeutische Maßnahme der Wundbehandlung angesehen werden. Sie ist kein Ersatz für ein sorgfältiges chirurgisches Wunddébridement, dass jeder Vakuumtherapie voraus gehen sollte, jedoch eine effiziente Alternative zur klassischen konservativen Wundversorgung. Obgleich in der Literatur vielversprechende klinische Ergebnisse der Wundbehandlung mit dem V.A.C.-System beschrieben werden, gibt es wenige wissenschaftliche Beweise, die einen positiven Effekt der V.A.C.Therapie untermauern. Der Erfolg zukünftiger Therapien wird von einem wachsenden Verständnis der pathophysiologischen Prozesse der Wundheilung unter Vakuumtherapie abhängen.

\section{Literatur}

${ }^{1}$ Barringer CB, Gorse SJ, Burge TS. The VAC dressing - a cautionary tale. Br J Plast Surg 2004; 57: 482

2 Bauer P, Schmidt G, Partecke BD. [Possibilities of preliminary treatment of infected soft tissue defects by vacuum sealing and PVA foam]. Handchir Mikrochir Plast Chir 1998; 30: 20-23

3 Bruhin A, Metzger J. [Principles of wound treatment]. Ther Umsch 2007; 64: 473-483

${ }^{4}$ Chen SZ, Li J, Li XY et al. Effects of vacuum-assisted closure on wound microcirculation: an experimental study. Asian J Surg 2005; 28 : 211-217

5 Chester DL, Waters $R$. Adverse alteration of wound flora with topical negative-pressure therapy: a case report. Br J Plast Surg 2002; 55: 510-511

${ }^{6}$ Deva AK, Buckland GH, Fisher E et al. Topical negative pressure in wound management. Med J Aust 2000; 173: 128-131

7 Fleischmann W, Lang E, Kinzl L. [Vacuum assisted wound closure after dermatofasciotomy of the lower extremity]. Unfallchirurg 1996; 99: 283-287

${ }^{8}$ Fleischmann W, Russ M, Westhauser A et al. [Vacuum sealing as carrier system for controlled local drug administration in wound infection]. Unfallchirurg 1998; 101: 649-654

${ }^{9}$ Genecov DG, Schneider AM, Morykwas MJ et al. A controlled subatmospheric pressure dressing increases the rate of skin graft donor site reepithelialization. Ann Plast Surg 1998; 40: 219-225

10 Gwan-Nulla DN, Casal RS. Toxic shock syndrome associated with the use of the vacuum-assisted closure device. Ann Plast Surg 2001; 47: 552-554

11 Jones SM, Banwell PE, Shakespeare PG et al. Complications of topical negative pressure therapy in small-diameter wounds. Plast Reconstr Surg 2004; 114: 815-817

12 Kaplan M. Abdominal compartment syndrome. Ostomy Wound Manage 2004; 50 : 20-21

13 Krasner DL. Managing wound pain in patients with vacuum-assisted closure devices. Ostomy Wound Manage 2002; 48: 38-43

${ }^{14}$ Mokhtari A, Petzina R, Gustafsson L et al. Sternal stability at different negative pressures during vacuum-assisted closure therapy. Ann Thorac Surg 2006; 82: 1063-1067

${ }^{15}$ Morykwas MJ, Argenta LC, Shelton-Brown EI et al. Vacuum-assisted closure: a new method for wound control and treatment: animal studies and basic foundation. Ann Plast Surg 1997; 38: 553-562

${ }_{16}$ Moues CM, Vos MC, van den Bemd GJ et al. Bacterial load in relation to vacuum-assisted clo- sure wound therapy: a prospective randomized trial. Wound Repair Regen 2004; 12: 11-17

17 Petzina R, Gustafsson L, Mokhtari A et al. Effect of vacuum-assisted closure on blood flow in the peristernal thoracic wall after internal mammary artery harvesting. Eur J Cardiothorac Surg 2006: 30: 85-89

${ }^{18}$ Schlatterer D, Hirshorn K. Negative pressure wound therapy with reticulated open cell foam-adjunctive treatment in the management of traumatic wounds of the leg: a review of the literature. J Orthop Trauma 2008 22: $152-160$

${ }^{19}$ Vermeulen H, Ubbink DT, Goossens A et al. Systematic review of dressings and topical agents for surgical wounds healing by secondary intention. Br J Surg 2005; 92: 665672

${ }^{20}$ Wackenfors A, Sjogren J, Algotsson L et al. The effect of vacuum-assisted closure therapy on the pig femoral artery vasomotor responses. Wound Repair Regen 2004; 12: 244-251

${ }^{21}$ Webb LX, Schmidt U. [Wound management with vacuum therapy]. Unfallchirurg 2001; 104: 918-926

${ }^{22}$ Weed T, Ratliff C, Drake DB. Quantifying bacterial bioburden during negative pressure wound therapy: does the wound VAC enhance bacterial clearance? Ann Plast Surg 2004; 52: 276-279

${ }^{23}$ White RA, Miki RA, Kazmier P et al. Vacuumassisted closure complicated by erosion and hemorrhage of the anterior tibial artery. J Orthop Trauma 2005; 19: 56-59

${ }^{24}$ Wild T, Otto F, Mojarrad L et al. [Vacuum therapy - basics, indication, contraindication and cost listing]. Ther Umsch 2007; 64: 495-503

25 Wild T, Stremitzer S, Budzanowski A et al. Definition of efficiency in vacuum therapy - a randomised controlled trial comparing with V.A.C. Therapy. Int Wound J 2008; 5: 641-647

\section{Univ.-Prof. Dr. med. Christian Krettek Direktor}

Prof. Dr. med. Tobias Hüfner

Leitender Oberarzt

Kirsten Sommer

Assistenzärztin

Unfallchirurgische Klinik

Medizinische Hochschule Hannover

Carl-Neuberg-Straße 1

30625 Hannover

krettek.christian@mh-hannover.de 